\title{
BONE GROWTH IN HEALTH AND DISEASE*
}

\author{
BY \\ EDWARDS A. PARK \\ Professor Emeritus of Pediatrics, Johns Hopkins University School of Medicine, Baltimore, Maryland
}

Before beginning my lectures I wish to record the pride and the pleasure with which $I$ received the invitation to come to Birmingham in order to inaugurate the Leonard Parsons Lectures, and, at the same time, to express my regard for Sir Leonard. $\mathrm{He}$ was a distinguished physician in the British classical tradition, an accurate observer and full of the wisdom derived from great experience and wide reading and salted by innate common sense. To this was added his keen human interest, understanding and a great kindness. His perspective was broad so that, when he considered a detail, he never lost sight of the whole. This enabled him to perceive relationships in their large proportions and to measure situations correctly and this also kept him from being diverted to the fashions of thought or predilections of the moment. He always remained consistent with himself. Whether in medicine or outside it, his judgment could be counted on to show sanity and reliability and in approaching the problems of others he was able to withdraw himself.

Sir Leonard had an easily awakened curiosity, and his nervous system must have been made of iron stuff and been under iron control, for in spite of the burden of his large practice and administrative duties he was able at will to disengage his thoughts from them and to turn to the problems of illness and to explore them. This ability always seemed to me remarkable; how few of us, carrying his responsibilities, would not be overwhelmed by them-too weary or absorbed to think beyond the attendant anxieties. His studies, which were always clinical and eminently practical, were characterized by breadth and soundness. Then, withal, he was simple, friendly and accessible and could adapt so easily to any situation, as when at my house he took off his coat and played baseball with the children and became one of them. It was these qualities of the heart which made people so love him.

In his chosen subject of paediatrics he was a world figure and elder statesman, a combination of intelligence, integrity and practical idealism, which characterize so many of your distinguished men. Though I saw him infrequently, I was, like others, drawn to him by his worth, and felt for him unstinted admiration.

The Scope of the Lectures

Prompted originally by a curiosity concerning the incidence of rickets, Dr. Richard Follis, Jr., and I examined systematically the bones of more than 1,500 children dying in the Harriet Lane Home between the years 1926 and 1942 from the various diseases and accidents to which young life is heir. My two lectures are chiefly based on observations made in the course of these studies. I regret that, since they represent our common labours and thoughts, they may not stand in his as well as in my name.

Inasmuch as these lectures concern bone growth, it is advisable to make certain basic facts clear at the outset. When bone is first laid down by the osteoblasts, it is in the form of osteoid; that is to say, the organic form of bone without the inorganic (Fig. 1A). The deposition of calcium salts in it is a later occurrence. A chief distinguishing feature of rickets is the abnormally broad encasement of the trabeculae with osteoid, which differs only quantitatively from the physiological, so far as is now known.

It is a common misconception to think that bone is destroyed by osteoclasts as opposed to osteoblasts. The osteoblasts form and also destroy bone; their action may go in either direction. The probable explanation of this double function is a reversible intracellular enzyme action. The osteoclast, which certainly has only a destructive function, is nothing more than an aggregate of osteoblasts. In their study of the reproductive cycle in the pigeon, Bloom, Bloom and McLean (1941) demonstrated an intense formation of medullary bone during the pre-ovulatory period, which passed abruptly into a correspondingly intense destructive phase during calcification of the shell of the first egg; this was followed by a return to normal within a few hours after the first egg was laid. In the pre-ovulatory

* The first of two inaugural Leonard Parsons Lectures delivered in Birmingham in 1953. 
period the reticular cells of the marrow changed into osteoblasts and the marrow cavity became literally filled with a network of bone lamellae as the result of their constructive activity. As calcification of the shell began, the osteoblasts aggregated into osteoclasts and then what had amounted to pure bone formation was reversed into pure destruction. After the first egg was laid the osteoclasts broke up into their constituent osteoblasts, preparatory to a repetition of the cycle. It is interesting that nature's device for furnishing the calcium needed to meet the emergency requirements for the shell should be to build a house of organic matrix, to fill it with calcium, and then to destroy the house in toto. In our studies of children, Follis and $I$ have repeatedly found evidence for the destruction of trabeculae by osteoblasts and for the formation of osteoclasts from osteoblasts. We have not found signs that osteoclasts broke up into osteoblasts or reticular cells. The conception of an osteoblastic-osteoclastic cycle was proposed years ago by Kölliker (1873).

\section{The Normal Growth Pattern in the Infant and its Determination}

When we first began our study of the bones of children, it never occurred to us that there could be any doubt regarding the normal pattern of growth. The essential criteria we assumed were regularity of architecture and completeness of calcification. If, then, the proliferative cartilage had an orderly arrangement in parallel columns, if the calcification of its provisional zone was perfect, if the trabeculae in their turn lay in an orderly vertically disposed network, and here and there were encased by mantles of osteoblasts with films of uncalcified bone beneath them, the so-called 'physiological osteoid', we considered that the postulates of the normal were fulfilled. It was some years before we realized that this picture failed to represent the normal in the infant and was even further removed in the case of the premature, with both of which our study was chiefly concerned, but was to be regarded rather as the modification of illness.

The very practical question then arose, how to determine the normal. In a number of our children death had occurred suddenly from illness of not more than one to three days' duration, too brief to alter materially the growth pattern, and in yet others it had happened in apparent health. Did the bones of these children furnish the normal pattern? The difficulty here was to be certain to exclude rickets which we had found roughly in 40 to $80 \%$ during the first two years of life (Follis, Park and Jackson, 1952). In some instances we were helped by knowing that vitamin $\mathbf{D}$ had been given in a large enough amount presumably to have prevented rickets, but our chief reliance was the evidence on histological examination that the maturation cycle of the cartilage cell had remained in full swing and the bottom-most layer of cartilage, ${ }^{*}$ that in contact with the shaft, was being actively invaded by capillaries and osteoblasts.

As all students of bone know, the cells of the proliferative cartilage undergo a maturation cycle preparatory, and essential to, their invasion by the shaft. The mother cells divide, their offspring divide, enlarge, become arranged as family groups in columns, swell further into the so-called hypertrophic cells, develop glycogen, presumably as a source of energy for the chemical process of calcification of the surrounding matrix (Gutman and $\mathrm{Yu}, 1950)$ and also phosphatase (Follis, 1949), and, soon after this has occurred, disappear, undergoing degeneration and death. In this terminal dissolution they evidently give off some substance which exerts a chemotactic influence on capillaries and osteoblasts, causing the capillaries to dilate and the osteoblasts to proliferate and grow big and which literally draws them into the empty capsules, to start the reconstruction of the cartilaginous matrix frame into shaft. In the histological section one could tell that the complete cycle had been occurring, if the end stage was there; namely, next to the shaft a zone of distended cartilage cell capsules, appearing empty or containing a shrunken palestaining cartilage cell, or one apparently breaking up or already broken. In many capsules, too, there were the capillaries and osteoblasts which had freshly penetrated (Fig. 2B). Now a cardinal feature of rickets, so far as the cartilage is concerned, is that this maturation cycle of the cartilage cell is interrupted by the disease, so that it does not go on to senescence and death. Therefore, the pale-staining, vacuolated-appearing zone of empty, or partially empty, cartilage cell capsules is never developed, and constantly found missing. If, then, examination of the cartilage shows maturation fully preserved, rickets could be excluded. Conversely, however, failure of the maturation cycle to develop did not mean that rickets was present, for under the influence of severe illness or malnutrition the cartilage may stop growing. Taking then the completed maturation cycle as the criterion, we were able to select from the large group of subjects of sudden death, or death after short illness, a smaller group showing the true pattern of growth. On these our description of the normal is based.

\footnotetext{
* Throughout the descriptions which follow the bone must be imagined as standing on end with the epiphyseal cartilage uppermost.
} 

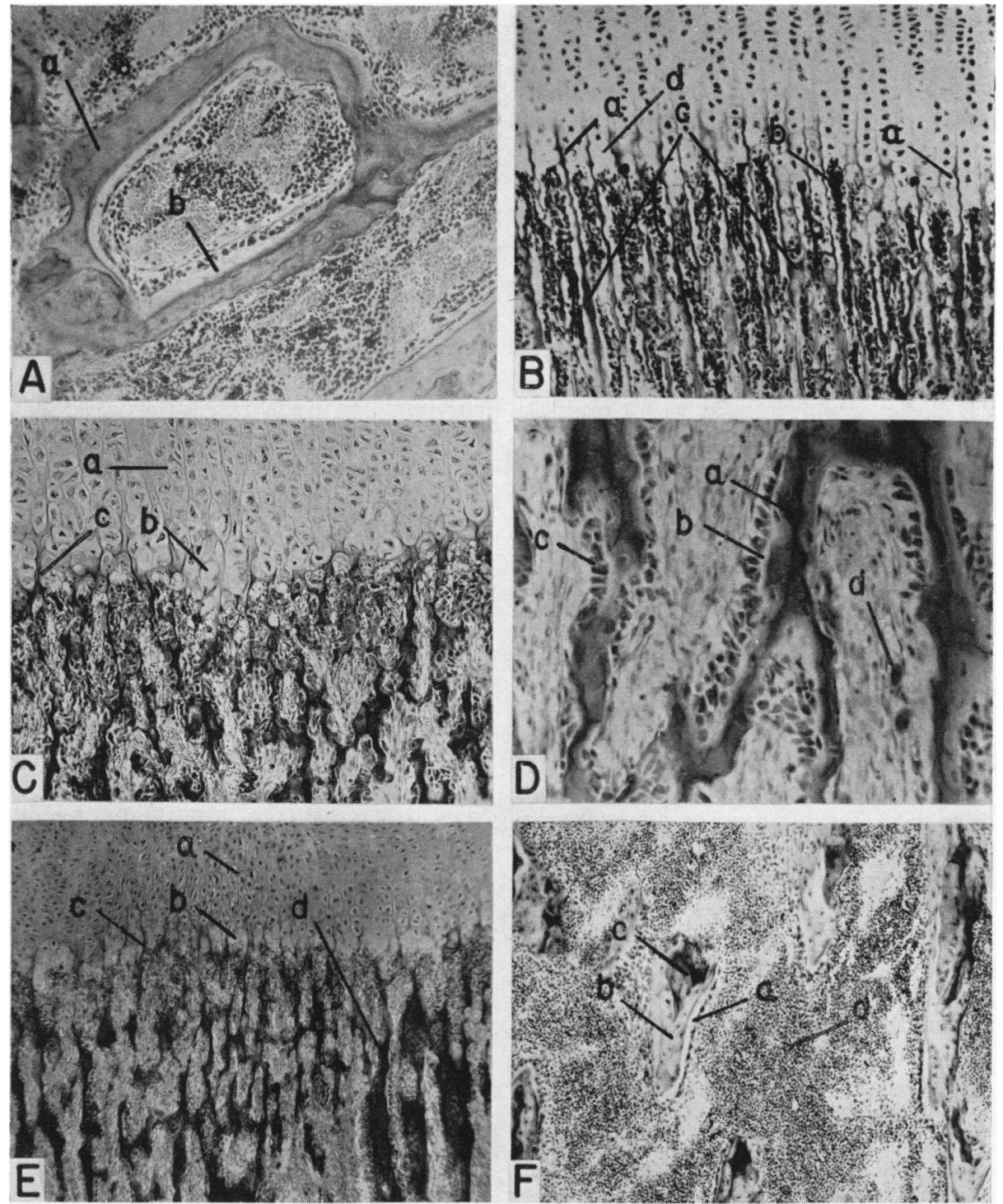

Fig. 1A.-Shaft in case of rickets to illustrate (b) osteoid; (a) calcified bone formed before the development of rickets. $\times 120$. Osteoblasts in orderly arrangement line the osteoid.

FIG. 1c.-Cartilage-shaft junction of normal prematurely born infant; (a) proliferative cartilage; (b) cartilage cell capsule empty because of death of cartilage cell at termination of maturation cycle; $(c)$ calcified matrix substance. $\times 60$. Note great irregularity of proliferative cartilage, and of trabecular network of shaft adjacent to it; also, intense proliferation of osteoblasts and paucity and irregularity of calcification of cartilage.

Fig. 1E. Cartilage-shaft junction of normal, full-term, 2-month-old infant, showing same irregularities exhibited by the premature infant in Fig. 1c, but in lesser degree. $\times 41$. (a) Proliferative cartilage; (b) empty capsules in process of being invaded by capillaries and osteoblasts, the criterion of normal growth; (c) calcified matiix substance extending into cartilage in advance of invading capillaries and osteoblasts; $(d)$ calcified trabecula.

FIG. 1B.-Cartilage-shaft junction of a normal young rabbit to show the orderliness of cartilage and of its invasion and transformation into shaft. (a) Calcified matrix substance extending in advance of capillaries and attendant osteoblasts; $(b)$ capillaries injected with india ink; $(c)$ trabecula in process of formation through the layering over of the calcified matrix substance with fresh deposits of bone

through activity of osteoblasts; $(d)$ empty cartilage cell capsules.

Fig. 1D.-High power view of Fig. 1C, to show the great numbers and size of osteoblasts. $\times 252$. (a) Core of calcified matrix substance of cartilage on which bone has formed; $(b)$ 'physiological' osteoid; $(c)$ the canopy of large osteoblasts; $(d)$ osteoclasts.

FIG. 1F.-Shaft of Fig. 1E $(\times 100)$ showing 'physiological' osteoid lined with osteoblasts; $(b)$ calcified bone; $(c)$ calcified matrix substance about which bone has formed; $(d)$ marrow. 
But before proceeding I must point out that our studies were focused on the costochondral junction of one of the middle tier of ribs, though in many instances ends of the long bones of the extremities were also studied. The reason for this was that in all necropsies we were able to obtain ribs but the other long bones only exceptionally. The ribs at their anterior ends grow the fastest of any of the long bones and consequently the cellular activities are greatest there; also the modifications from illness reach their most extreme development. At the ends of all the long bones the growth pattern is in general principle the same, but varies greatly in intensity with the rate of linear growth at the bone end in question. If linear growth is slow, as for example at the upper ends of the radius and ulna, the activities of both cartilage cell and osteoblast are greatly reduced. Our description essentially applies to the costochondral junction.

\section{Histological Picture in the Premature Baby}

In the healthy premature baby the proliferative zone of cartilage is extremely irregular (Fig. 1C). The cells in certain areas, particularly at the sides, may be in columns, but in general they are in thick, irregularly arranged fascicles or are jumbled together in no apparent order. Calcification extends into the cartilage scarcely a single cell deep and, where it has occurred, is so light that it appears as defective as in beginning rickets. One of the most striking features is the zone of hypertrophic cartilage cells three to five deep and below it the pale zone of distended capsules in process of being penetrated by the capillary-osteoblast complexes.

Turning now to the shaft, the arrangement of the trabecular network next to the cartilage is quite as disorderly as the cartilage itself, which is understandable, since its architectural plan is always derived from the cartilage: malformed cartilage, malformed trabeculae. The osteoid borders of the trabeculae are in places as thick as those found in moderate rickets. Where active growth is occurring, the surfaces of the trabeculae are covered with great numbers of osteoblasts. As seen silhouetted along the edges of the trabeculae, as when the section passes through their middles, the osteoblasts are oblong or square with large pale-staining nuclei and large vacuoles and are separated from the calcified bone by the layers of osteoid which they have just formed (Fig. 1D). When the section happens to cut across the surface of a trabecula tangentially, one can see that the osteoblasts form a perfect mosaic, cell lying against cell, making a complete covering. One of the most striking features is the relatively giant size of the osteoblasts.
As already pointed out, Bloom and his collaborators disclosed examples of pure growth and pure destruction of bone in the female pigeon at different phases of the reproductive cycle. Probably nowhere in mammalian life, certainly not in the child, does pure growth or pure destruction occur, although one or the other may happen, or can be made, experimentally, to predominate. The two processes are so interrelated that one never occurs without the other. In the young healthy adult bone destruction must match bone formation; in the aged destruction must imperceptibly predominate; in the rapidly growing child formation must exceed destruction, but only by the balance of the new bone which survives. In these rapidly growing bones of the premature infant destruction of the trabeculae was almost as prominent as new bone formation.

Incidentally, the histological picture in the premature infant furnishes a most vivid illustration of his predisposition to rickets. One can truthfully say that in his accelerated growth he continually moves along the verge of it. All that is needed for rickets to supervene is for the calcification rate to drop behind growth rate or, the other way round, for growth to exceed its power to keep up.* The studies of Gordon and McNamara (1941), Clifford and Weller (1948), Benjamin, Gordon and Marples (1943), Wright, Filer and Mason (1951) have shown how inefficient the absorption mechanisms for calcium, fat and the fat-soluble vitamins of the premature infant are.

\section{Modifications in the Full-term Infant}

In the full-term infant in the bloom of health, the histological picture does not differ greatly from that of the premature except that cell growth both in cartilage and bone seems to be occurring not so rapidly and with more order and the borders of physiological osteoid are not so broad (Fig. 1E).

In the older child, growing at a considerably slower pare, the zone of proliferative cartilage is less deep and its pattern and that of the trabecular network of the shaft are more regular and the calcification of the provisional zone heavier. But the pale zone of distended capsules at the shaft border, the osteoblasts and capillaries which have penetrated into them, the mantles of osteoblasts covering the growth surfaces of the trabeculae, and the films of physiological osteoid are all there.

\footnotetext{
* It is perhaps a question of academic interest whether the condition just described in the bone of the premature infant with its scant calcification of the cartilage and its broad osteoid borders ought not to be called rickets. If so, it is a physiological rickets, and not vitamin $D$ deficiency rickets, for the picture occurs under Vitamin D administration.
} 
The Effect of Severe Illness on the Normal Growth Pattern

I must give some notion what is meant by severe illness. In our series of children, as already pointed out, there was a wide variety of illnesses, some lasting only for hours, some protracted, and all severe in the sense that they ended fatally. Random causes of death were burns, fatal operations, in particular for hydrocephalus, mechanical conditions such as intussusception and other forms of intestinal obstruction, haemorrhage, malnutrition states, scurvy, rickets, prematurity, syphilis, vomiting and diarrhoea with dehydration and acidosis, infection, etc. In the great majority of instances the cause of death was infection, e.g., pneumonia, meningitis, septicaemia, dysentery, etc., and these were ordinarily of a most severe nature. We were, of course, able to classify the conditions responsible for the death of the children according to duration, but would like to have divided them into nutritional and infectious. But clear separation we found impossible for the children suffering from nutritional disorders usually died of terminal infections, and children having severe infections usually suffered nutritionally. What we mean by severe illness then is severe infection of 10 days' or more duration and also severe malnutrition from any cause, for the effects of both on bone growth from the histological point of view seemed to be the same. In the last analysis the action of severe infection on the bone cells probably has a nutritional basis.

What happens when severe illness supervenes in the child growing at this rapid pace? First, I describe the changes in the shaft (Fig. 2A). There new bone formation has virtually stopped. The mantles of thick osteoblasts have gone, we are not sure where. Many have fused with their neighbours into syncytial formations and become osteoclasts (Fig. 2B). We have looked for changes which might indicate that the osteoblasts have disintegrated but have found little evidence of nuclear or cytoplasmic debris. The question must be settled by experimental procedures. The absence of such change has puzzled us, for, if the great numbers of osteoblasts, packed together over the areas of active growth, had been produced by division and were fixtures there, so to speak, one would expect to find evidence of their disintegration in order to explain their disappearance. But mitosis is seen but rarely, even when osteoblastic proliferation is at its peak. Bloom and his collaborators have called attention to its rarity in the osteoblasts of the pigeon during the period just before the shell formed, at a time when they are literally filling the marrow cavity with bone. For that reason we have wondered if the surfaces of the trabeculae had lost their chemotactic action and the osteoblasts, changing over into reticular cells, had moved away from them. In some instances, in which the illness was brief, it looked as if this spreading movement away from the trabeculae might be occurring, for particularly in the interspaces just under the cartilage evidently metamorphosing osteoblasts were spread out in an even network much as in scurvy. There is no doubt that when this regressive change in the lining osteoblasts is occurring, the trabecular surface loses its power to hold them tightly. When actively forming bone, they lie close against the surfaces, but in the transition states they tend to lie along, rather than close against, the surfaces, and at the same time their formation becomes irregular. But the disappearance may be more apparent than real, for the shrinkage of the cells and their transformation into types difficult or impossible to distinguish from ordinary connective tissue may make it easy to underestimate their numbers and to think they are not there. In extreme cases one finds little in addition to the sparsely cellular endosteal membrane.

As seen in the histological preparations of the ribs and long bones of malnourished children from which the marrow cells had largely disappeared, the endosteal membrane is a very definite structure, composed of flattened cells forming a complete sheet covering the trabecular surface. In sections cutting the membrane across, so that it is seen silhouetted along the trabecular edges, the nuclei appear long and thin and the cell bodies long and spindle shaped. They lie close to the bone surface, often so close that it is difficult to see them and even to be certain that they are there. When the plane of the section happens to pass tangentially through the membrane, so that one can look down on it from above, so to speak, the nuclei appear oval or round, fairly large and more numerous than anticipated from the cut edge (Fig. 2A). Evidently the reduction in size is largely in the thickness of the cells. The proof, however, that they form a complete membrane is evident in sections passing through the middle of longitudinally directed trabeculae because under favourable conditions the membrane can be seen to form a continuous line. However, the best demonstration is furnished where the membrane has chanced to have been pulled away from the bone surface in the process of cutting (Fig. 2c). Then its edge can be plainly seen to form a line, bounded by the marrow on one side and, on the other side, by the clear space created by the tear. Since this membrane is found on every trabecula, it must be conceived of as continuous, covering the entire interior surface of the bone, including the entire trabecular system, as 
the peritoneum does the viscera. However, where bone is being destroyed, it has broken up, leaving the surface bare and directly exposed to the osteoblasts and osteoclasts engaged in eroding it (Fig. 2B). In some instances in which atrophy has reached an extreme development we have been unable to identify the membrane over considerable areas of the trabecular surface, which appeared to be in direct contact with the marrow, although not being actively destroyed.

Destructive activity had dwindled also, as would be expected if it depended, as it does, on the presence of the osteoblastic group of cells. However, when one examines the trabecular edges with care, one can always find scattered areas of destruction, character- ized, as indicated above, by disintegration of the endosteal membrane and the presence of an osteoclast or two and a few osteoblasts. But the osteoclasts, like the osteoblasts, are small and inconspicuous. So surface disintegration appears to be moving forward, even though at a reduced rate. But it is most interesting to note that even single cells of the endosteal membrane can dissolve the bone surfaces immediately under them, for where they touch, the surfaces have been hollowed out into little scallops (Fig. 2D). This semi-cryptic surface destruction of the trabeculae, going on without compensating new bone formation, must be responsible for the extreme degrees of rarefaction reached when a wasting disease is prolonged, though the
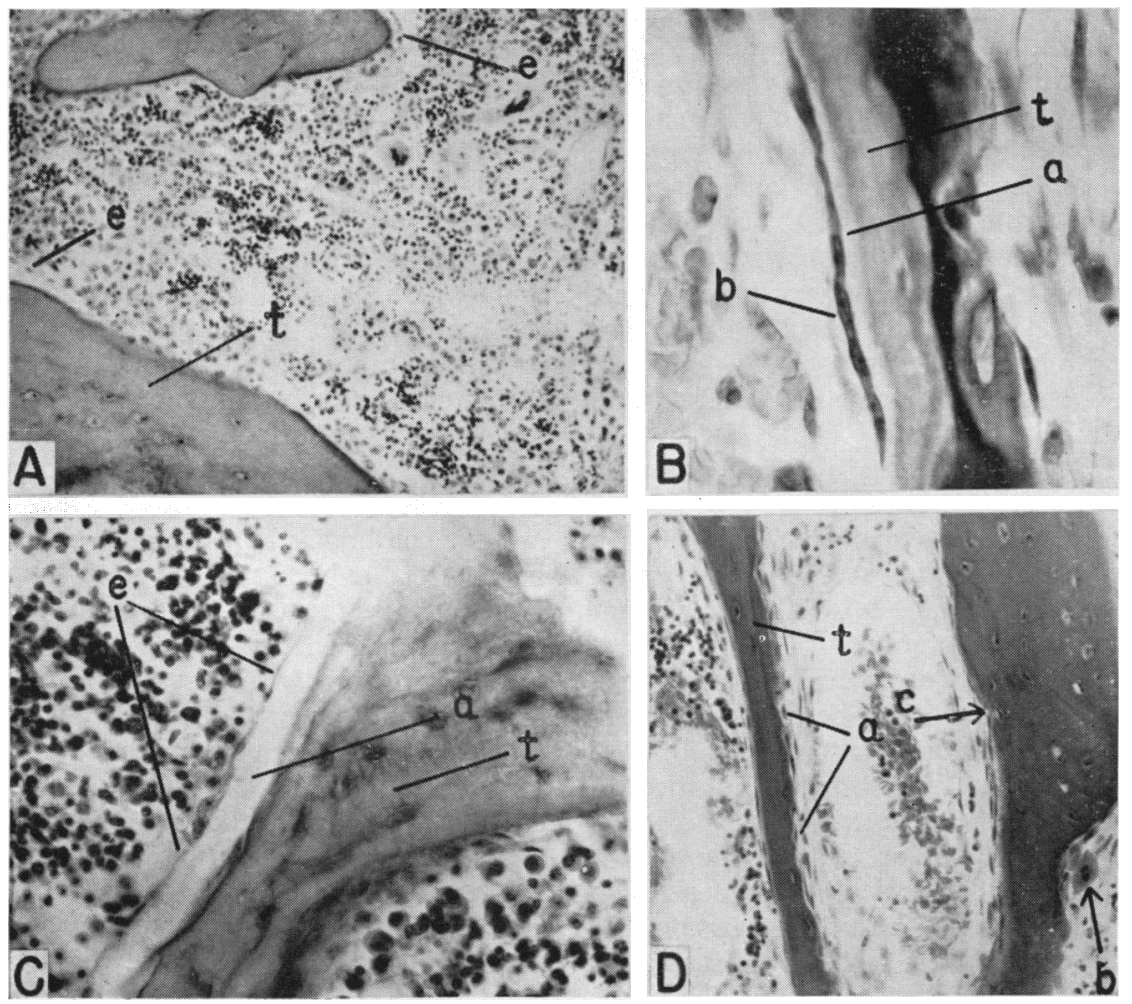

Fig. 2A.-Trabeculae of rib from 13-months-old child, dying after protracted tuberculosis. $\times 120$. At first glance the trabeculae $(t)$ seem devoid of covering cells but in two places, where soft tissues have been pulled away in trauma of cutting, there is completely intact endosteal membrane (e).

Fig. 2c.-Trabecula from rib of 6-months-old child dying of tuberculosis $(\times 518)$; $(e)$ endosteal membrane which had been pulled away from the bone surface in the process of cutting and in consequence is clearly visible; $(t)$ trabecula showing osteocytes; $(a)$ clear space produced by tear.
FIG. 2B.-Trabecula, rib, from well nourished 2-months-old child, dying of pneumococcal septicaemia and meningitis of eight days' duration $(\times 518) ;(b)$ osteoblasts in regression; they have fused, the beginning of osteoclast formation; ( $a$ ) osteoid; (t) calcified bone. The presence of the fairly broad osteoid makes it necessary to think that before the acute illness the osteoblasts formed, as in Fig. 1F, a canopy of orderly, well developed cells.

FIG. 2D.-Trabecula $(\times 120)$, rib of 11-months-old child dying of intussusception, showing active surface destruction by $(a)$ single osteoblasts which have produced scallops; $(b)$ osteoclast with a company of osteoblasts destroying trabecular surface; $(t)$ bony trabecula; $(c)$ the lacuna occupied by an osteocyte, which has been uncovered by osteoblasts acting as single cells. Osteoblasts are in extreme regression with reversal of function. 
qualifying statement should be made that in many instances the destruction may be marked.

But it must not be thought that the changes, such as we have described in the shaft, and are about to describe in the cartilage, have always been found in severe illness. In some instances growth activity has been continuing in both cartilage and shaft as if the illness had not occurred. In some of the premature infants it has been extraordinary to witness how growth of both the cartilage and the shaft had proceeded full blast to death itself. The preservation of the normal growth activity of the bone in the face of the most damaging environmental conditions is no more remarkable than the preservation of the nutrition, which often seems not to be affected at all. As a matter of fact the nutritional state of the child as a whole and the size and activity of the bone cells often seemed to correspond.

In this human study we have not mentioned the circulation for the reason that we have found it extremely difficult to estimate, probably largely because of the disturbances in it at the time of death.

\section{Transition States}

I have alluded to transition states from extreme growth activity to cessation under the influence of severe illness, but have not the time to trace them in detail. In some instances the regressive change can be noted as early as the third day and it may reach the extreme development described certainly by the middle of the second week.

\section{The Effects on the Cartilage}

In the proliferative cartilage also one finds signs of growth failure. If growth stops, the maturation cycle of the cartilage cell naturally is halted. Since on the one side the mother cells no longer divide and, on the other side, all the already fully developed cells, in a state to be invaded and destroyed by the capillaries and osteoblasts, have been removed, the cartilage plate, as seen in the histological section, becomes reduced to a thin band. In this band calcification becomes progressively heavy, for the very reason that the cartilage is dormant and calcium salts are being continually adsorbed as on the surface of a crystal. In its most extreme development the picture simulates that seen at the expiration of normal growth, the stage preceding epiphyseal closure (Fig. 3A). Then calcification becomes so profuse that it extends all around the cartilage cells so that the latter, as seen under the microscope in haematoxylin- and eosin-stained sections, seem to peer out of the dark blue background of calcified matrix like eyes. As already indicated, complete stoppage of growth in the infant for any long period, even under most severe wasting illnesses, has proved rare. Usually the picture has been rather that of slowed growth. Evidently the growth power of the cartilage is difficult to suppress over any length of time.

What happens when growth of the epiphyseal cartilage is merely slowed I shall postpone till I discuss lattice formation: I now turn to the curious transverse stratum formation which results from greatly slowed, or completely arrested, growth of the cartilage.* Since the stratum shows in the x-ray film as a 'transverse line', it has awakened clinical interest. I shall make clear the mechanism of its development as recently studied experimentally (Park and Richter, 1953), not going into the natural history which has been excellently presented by Harris(1933).

\section{Mechanism of Development of Transverse Stratum ('Transverse Line') Formation}

It will be easiest to understand the formation, if one supposes that growth of the cartilage almost stops and all the underlying cancellous trabeculae have been resorbed, a condition easy to produce experimentally in the rat, either by limitation of diet or hypophysectomy. The cartilage plate can then be imagined as topping the shaft like the cover of a jar. The osteoblast has no architectural ability; it cannot create new patterns; all it can do is to settle on pre-formed structures natural to it and form bone on their surfaces. Such pre-formed structures are the frame of matrix substance of the cartilage, and the surfaces of the trabeculae or lamellae of bone already formed as the result of previous osteoblastic activity. The shaft of course elongates because the cartilage, as it grows, keeps producing matrix frame on which some of the osteoblasts keep settling and turning into bone as fast as their fellows lay it bare by destroying its contained cartilage cells. The reason that the trabeculae of the shaft are arranged in the main longitudinally is because the matrix frame, on which they settle and operate, has the longitudinal arrangement (Fig. 3c). Indeed, as already pointed out, the pattern of the cancellous tissue of the shaft immediately under the cartilage is the pattern of the matrix frame and often from this pattern one can tell what the pattern of the future trabeculae will be. When, therefore, growth of the cartilage has stopped, the osteoblasts, unable to grow into it, have no alternative except to settle on the bare undersurface of the epiphyseal plate

\footnotetext{
* In discussing transverse stratum formation resulting from partially or completely arrested growth, I am not referring to the strata of increased density produced by lead poisoning, white phosphorus administration, bismuth administration, etc. The mechanism of their development is entirely different.
} 

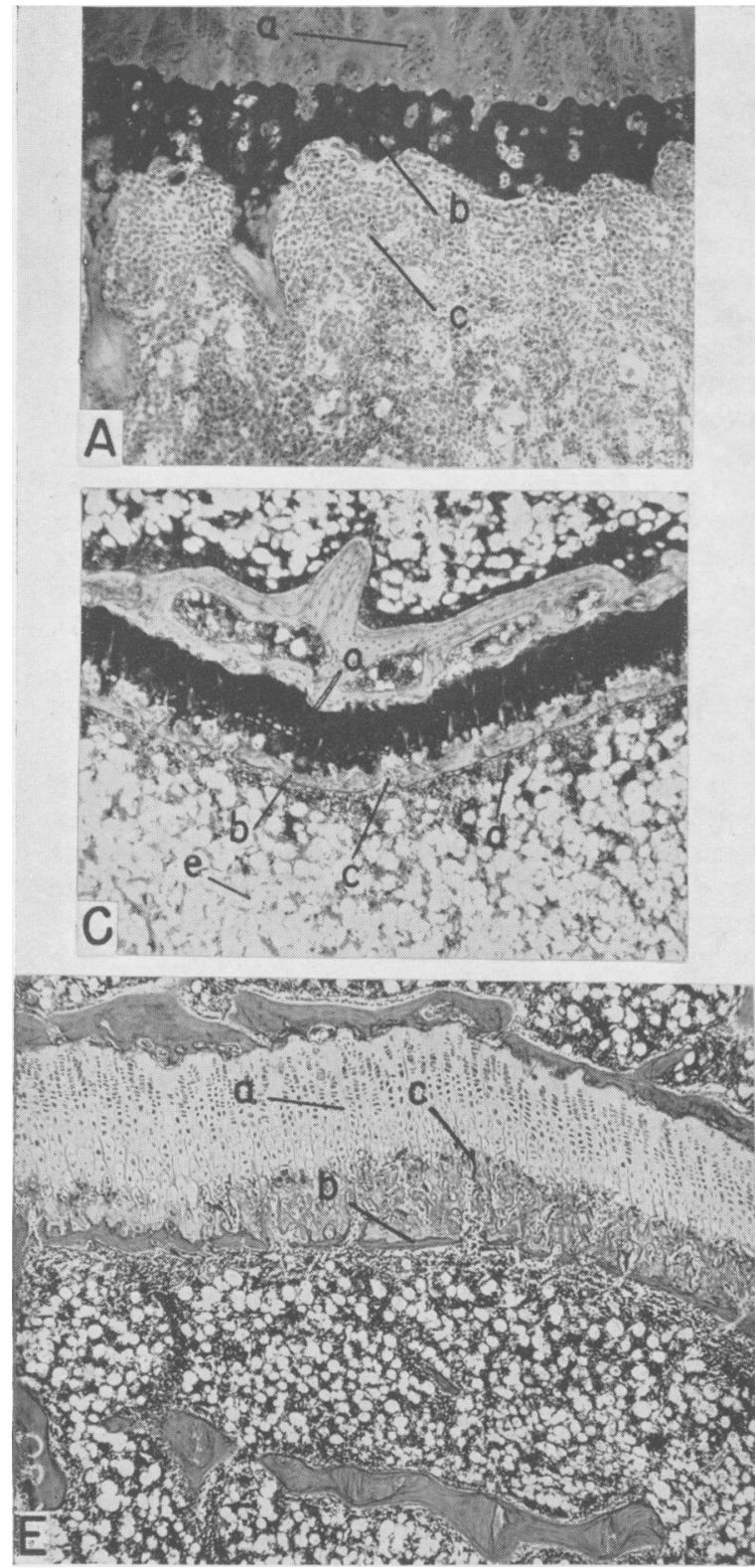

FIG. 3A.-Upper humerus from 20-months-old child dying of extensive tuberculosis. $\times 66$. The section shows extreme slowing of growth, amounting to arrest. The calcification of the cartilage $(b)$ has become almost confluent; $(c)$ marrow.

FIG. 3c.-Upper tibia of a domestic rat, aged 111 days, kept on diet of dextrose and vitamin $B_{1}$ for 49 days, followed by stock diet for five days. $\times$ 40. Proliferative cartilage during the dextrose- $B_{1}$ period reduced to a thin band. Basic transverse stratum of bone formed during the dextrose- $B_{1}$ period is undergoing thickening with resumption of growth due to change to stock ration. (a) Proliferative cartilage; (b) transverse stratum; (c) hole admitting passage of capillary with accompanying osteoblasts; $(d)$ osteoblasts forming bone on under surface of stratum; $(e)$ marrow of shaft heavily laden with fat ; $(f)$ epiphysis.

FIG. 3E.-Upper tibia of domestic rat, aged 140 days, on a diet of dextrose and vitamin $B_{1}$ for 58 days, stock ration for 13 days. $\times \mathbf{4 0}$. (a) Proliferative cartilage has now reached full development as indicated by its vertical columns, ending in the zone of empty capsules at the junction with the shaft. The transverse stratum $(b)$ has been penetrated and passed by the wave of $(c)$ vertically invading capillaries with their attendant osteoblasts. Trabeculae are now being formed again in vertical direction, as under normal conditions. When the capillaries and osteoblasts have advanced through the stratum the thickening process ceases.
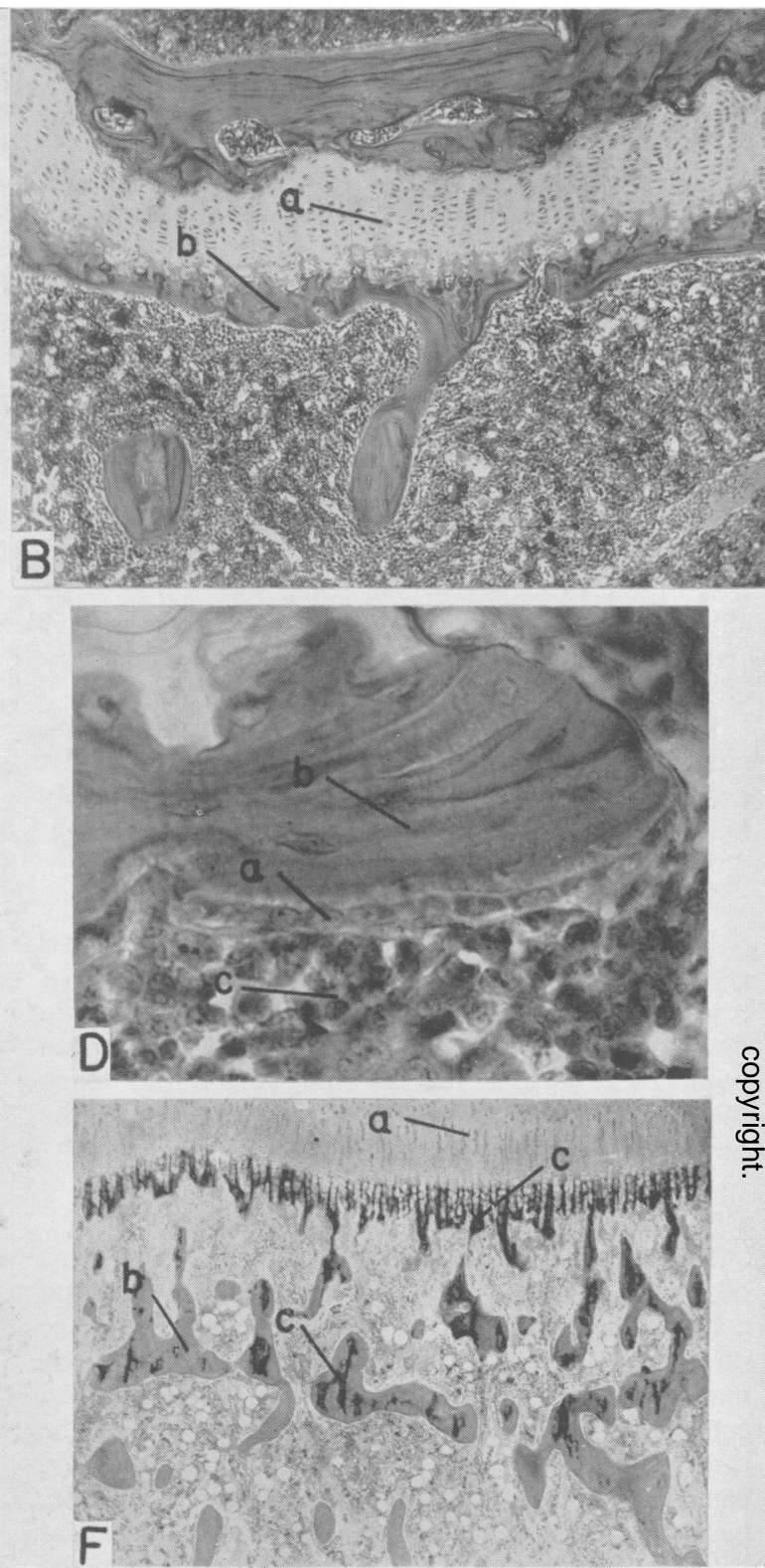

윰 흘

Fig. 3B.-Upper tibia of a wild rat, unknown age, 68 days after hypophysectomy, $\times 64$. The basic stratum of bone $(b)$ lining the under surface of the greatly narrowed proliferative cartilage $(a)$ is well shown. The basic stratum, formed during growth recession, is much better developed than usual.

FIG. 3D.-Upper tibia of domestic rat, aged 137 days, on a diet of dextrose and vitamin $B_{1}$ for 72 days, stock ration for eight days. $\times$ 488. (a) Lining osteoblasts forming new bone on the under surface of the $(b)$ basic stratum; the pale space between the osteoid and bone is caused by the freshly formed osteoid; $(c)$ marrow.

Fig. 3F.-Upper humerus of a 15-months-old child, dying of pneumonia after having recovered from dysentery $7 \frac{1}{2}$ weeks previously. $\times$ 13. (a) Proliferative cartilage; (b) transverse stratum of arrested growth, presumably formed during the dysentery, as it appears in single plane of histological section. It is composed of bone with (c) inclusions of calcified matrix around which the bone formed; bordering the cartilage at the junction with the shaft is the provisional zone of calcification with its subjacent thicket of calcified matrix on which bone is beginning to form, but is not visible with the magnification employed. 
and form bone upon it and, since this is horizontal, the bone which they form is horizontally disposed. This horizontal sheet of bone, formed as growth of the cartilage slows and stops, is the basic transverse stratum (Fig. 3B). Indeed, this basic stratum alone, formed during the recession of cartilaginous growth, may be responsible for a well developed 'transverse line', exactly as when growth of the epiphyseal cartilage stops altogether, preparatory to closure of an epiphysis.

But it is most important to realize that when growth of the cartilage resumes, the stratum undergoes its thickening, i.e., ordinarily the phase of recovery is the one in which the horizontal stratum chiefly develops its thickness. Years ago, when he was experimenting with vitamin A deficiency in the rat, Wolbach fully realized that the basic stratum formation occurred during the slowing of growth of the cartilage and that its thickening was a recovery phenomenon, though he did not discuss this at the time.* The explanation of the thickening, when growth of the cartilage resumes, is as follows. When growth is resumed, the cartilage cells at once begin to divide but are obliged to complete their maturation cycle before it is possible for the shaft forces, the capillary-osteoblast complexes, to invade them. They cannot penetrate until the columns have formed and also their lowermost cells have degenerated and died, and this stage is not reached until the cycle is completed. The completion of the maturation cycle of the cartilage requires time, since it involves division, maturation, arrangement in columns, senescence, and finally death, as already explained. In this lag period the osteoblasts, which are able to form bone immediately, cover the undersurface of the basic horizontal stratum with new layers (Figs. 3C and D). This thickening continues until the maturation cycle of the cartilage cell has been completely re-established and longitudinally disposed columns of cartilage cells once more have formed with longitudinally disposed matrix framework. Then the capillary-osteoblast complexes break through the stratum into the cartilage columns beyond, and the process of trabecular formation in the longitudinal direction is resumed (Fig. 3E). With the passage of the wave of active growth through the line into the growing cartilage beyond, the thickening of the line comes to an end.

For the purpose of making clear the cellular mechanics of transverse stratum formation we assumed that growth of the cartilage had stopped entirely and its undersurface had been rendered trabecula free. But, as transverse stratum formation

\footnotetext{
* Personal communication.
}

ordinarily occurs in the child, the underlying trabeculae have still remained intact. However, the principle of formation is exactly the same as that described. With the slowing or arrest of longitudinal growth the osteoblasts cover over the under surface of the cartilage in the horizontal direction with the basic bone film, knitting the trabeculae together at their bases and with it the under part of the matrix frame. Then when growth activity is resumed, they thicken the stratum. In the transverse strata of the bones of children, which have been a common finding in the studies by Follis and myself, one can see incorporated in the substance of the stratum the patterns of the bases of the trabeculae as they were at the time when the line formed (Fig. 3F).

In conversation Dr. John Caffey once expressed doubt that transverse 'lines' in bones could always depend on growth inhibition, for he had observed in children, one of whose limbs was growing faster than the other, that the thicker line was in the faster growing bone. Since the thickening of the line, except at the end of the growth period, occurs chiefly during recovery, a proportionately greater thickness would be expected in the more rapidly growing bone.

Of course the reason that the horizontal stratum of increased density appears as a line in the x-ray film is because it is caught on edge. Actually, when seen in its three dimensions, its formation is like a heavy rope net strung horizontally across the bone. The net-like structure is due to the fact that it is full of holes, where the blood vessels and osteoblasts have broken through, and the bounding cords are the interlacing horizontally disposed trabeculae (Fig. 4A). The transverse stratum once formed of course never moves (Lacroix, 1951). The reason that it becomes increasingly separated from the cartilage with the passage of time is of course that the cartilage keeps growing away from it and new normal shaft continues to be formed on the epiphyseal side.

\section{Mechanism of Development and Significance of the Growth Retardation Lattice}

As seen in histological section in single plane, it is a framework of calcified cartilaginous matrix in the interstices of which are large numbers of cartilage cells (Fig. 4B). It has the pattern of the cartilage for the reason that it is nothing other than calcified cartilage. In normally growing cartilage the only part which is calcified is the provisional zone of calcification, i.e., the lowermost layer bordering the shaft, and this is only two to three cartilage cells deep. But in the condition I am describing, the lattice, which is calcified in its entirety, may be as 
long as the epiphyseal cartilage itself. The gross abnormal feature is the existence of this large formation of calcified cartilage at the top of the shaft which should not be there at all. But the formation has special peculiarities. The matrix framework is heavy and the vertical limbs of it are joined to their neighbours by successions of horizontal cross bridges. Moreover, the interstices in the framework are narrow so that the vertical limbs lattice, if life had continued-are shrivelled in contrast to the perfect round or oval contour of the nuclei in the lattice immediately below. In the lower one-third or so of the lattice the cartilage cells have been removed or are in the process of being, and there one finds the removers, namely the capillaries and ancillary osteoblasts. But these invading complexes of vessels and cells are found only here and there and extend upwards into the lattice
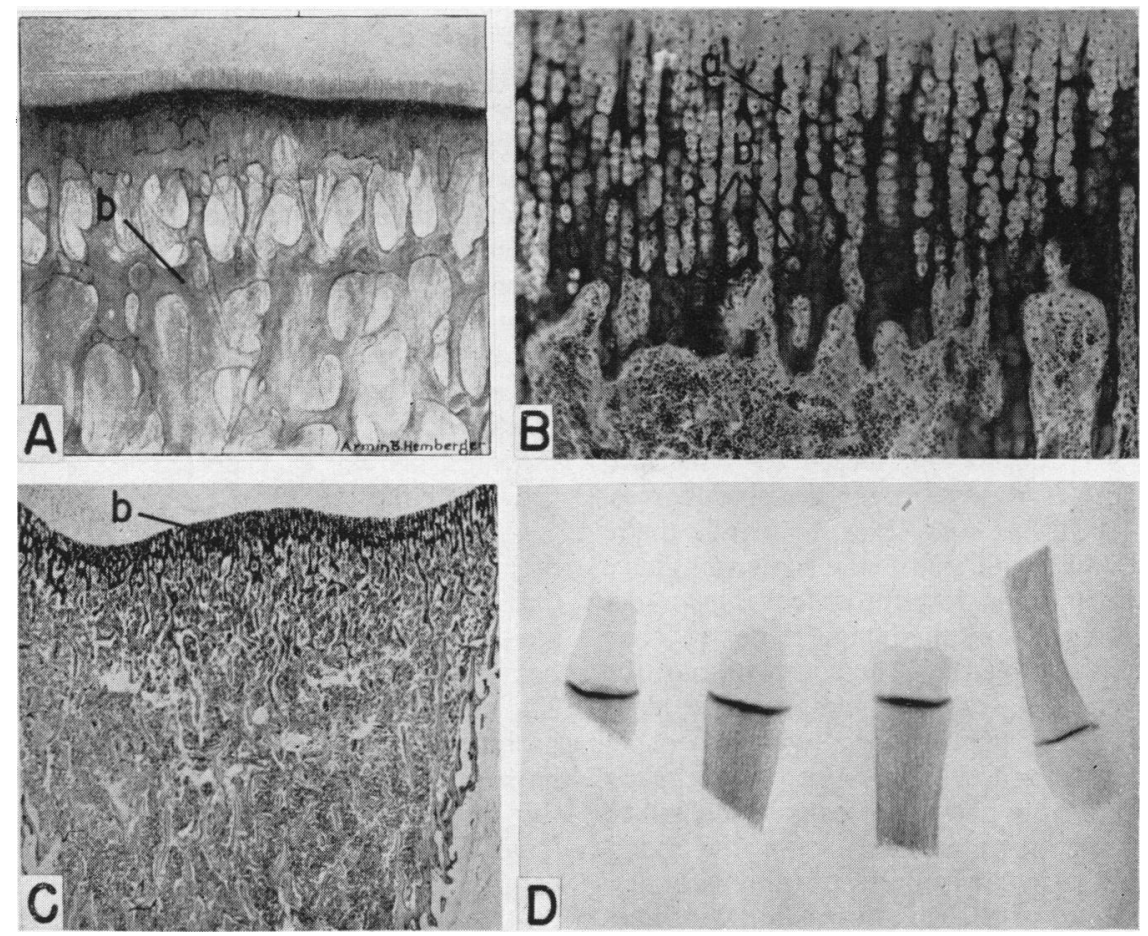

FIG. 4A.-Drawing of a transverse stratum, showing its formation in three dimensions (see text).

FIG. 4C.-Rib of 3-month-old child, dying of staphylococcal pneumonia and having also obstruction of the pancreatic duct with extreme emaciation. $\times 6 ;(b)$ the lattice, composed of calcified matrix framework, appearing black.

FIG. 4B.-Rib of 18-month-old child, dying of pertussis and bronchopneumonia of four weeks' duration. $\times 50$. The lattice $(b)$, representing the framework of calcified matrix, is beautifully shown with its (a) contained cartilage cells.

FIG. 4D.-X-ray film from Fig. 4C showing shadow cast by lattice.

lie close to each other. Also its calcification is particularly heavy and includes the horizontal bridges. The quantity of cartilage cells in the matrix frame varies. Perhaps on the average they occupy the upper two-thirds. They have round or oval, rather faintly staining blue nuclei and the cell bodies are clear and so pale that they look almost white, furnishing a striking contrast to the blue-staining cells immediately above. Curiously, in many instances, the nuclei in the cartilage cells immediately above the calcified part-the layer of cartilage which would have calcified next and become a part of the

irregular distances. Osteoblasts can usually be discovered in the lacunae, formerly occupied by the cartilage cells, and often they appear to be filling these with bone. They seem to pick out the most secluded lacunae, those lying at the outermost fringe of the capillary distribution; along the main tracks of invasion one finds here and there thin films of bone, but frequently none. But the lattice is essentially composed of the calcified matrix substance of the cartilage with only a small admixture of bone, and of course in the larger part of it, since that is occupied solely by the cartilage cells, there is none. 
An additional feature of great importance is that in the shaft, in these cases of extensive lattice formation, the osteoblasts are invariably found in a state of extreme atrophy.

What is the explanation of this over-development of calcified cartilage? The reason is obvious. The osteoblasts have been either so reduced in number or rendered so impotent that they have been unable to perform their function of destroying the cartilage cells, thinning out the lattice and building bone.

The failure to thin out the lattice requires a word of explanation. I emphasized at the beginning that the osteoblasts possess two opposite functions, creative and destructive. Normally, at the moment when they destroy the cartilage cells at the cartilageshaft junction, they begin to form bone on some parts of the matrix frame and destroy other parts. In order to make my point clear, suppose that, if just under the cartilage-shaft junction they have succeeded in destroying perhaps one-half of the framework, a little farther down in the shaft two-thirds will have been destroyed, and still further down, nine-tenths. The parts which survive are presumably those which are essential structurally and protected because they are stimulated by stress and strain; the ones which are destroyed are, again presumably, those which are structurally useless or become so with increasing linear growth. Those that remain become increasingly thick as the result of being covered repeatedly with fresh layers of bone. This selective destruction of the trabeculae of the lattice is what I meant by thinning. The persistence of the lattice, virtually in toto, is much more striking evidence of the failure of destructive function than is the paucity of the bone formation on it evidence of constructive action. The inability of the osteoblasts to attack the cartilage and deal with it immediately, as fast as it is produced, is actually the same phenomenon as the one so evident on the surfaces of the trabeculae lower down in the shaft, where the osteoblasts seem to have relapsed into complete futility. The way then that the formation develops is that the cartilage keeps growing, i.e., producing more and more of itself, the product keeps calcifying and the whole, remaining intact, keeps accumulating in ever increasing amounts in front of the shaft. In transverse stratum formation the fault is failure in growth of the cartilage; in the growth retardation lattice it is in osteoblastic function.

In the nutritional injuries caused by severe illness the cartilage cells like the osteoblasts shrink, and at the same time the amount of matrix substance which they form is increased. The reason that the matrix frame appears so thick then is because it has been produced in excess and the reason that the inter- stices of the lattice are narrower than normal is because they represent the spaces occupied by the shrunken cartilage cells. Calcification is so heavy because, with the slowing of growth, calcium salt deposition in the matrix had time to augment by adsorption. The reason for the cross bridging is that the cross bridges of matrix, which are nothing more than the horizontal partitions of the matrix frame separating the cartilage cells from each other, plus the walls of the capsules above and below, have not been carried away by the capillaries and osteoblasts, owing to the suspension of invasion. The reason that the cross bridges are so heavily calcified is because time has allowed the calcium salts to extend into them.

For some time Follis and I believed the reason that the lattice was not invaded was because its heavy structure, and in particular cross bridging, formed a mechanical barrier. These do interpose a mechanical barrier as soon as the normal growth processes become re-established. Then the vascularosteoblastic complexes make detours in order to get into the lattice where they can do so most easily and create broad vertically running paths of destruction often quite far separated from each other. The result is often a destruction of the lattice piecemeal so that chunks of it are isolated and left behind. The osteoblasts working their way into these chunks fill them with bone, converting them into giant trabeculae.

The growth retardation lattice has a similar origin to that of the metaphysis in rickets. But in rickets the fault lies in the impenetrability of the cartilage, not in the impotence of the osteoblasts. The lattice in scurvy develops in identically the same way as the result of an absolutely complete inactivation of the osteoblasts, whereas cartilaginous growth is able to continue. In scurvy the lattice remains completely free from any bone formation with the result that it fractures and shivers into fragments, while in the growth retardation lattice there is sufficient bone formation to prevent fracture.

The reasons that the growth retardation lattice casts a dense shadow in the $\mathrm{x}$-ray film is clear enough; the density of the shadow is due to the density of the thicket of heavily built, compact, heavily calcified matrix frame (Figs. 4D and E). I have often been asked by younger members of the staff to look at $\mathrm{x}$-ray films of young children because of a shadow at the end of a long bone which suggested lead poisoning. It was evidently not that, but was growth retardation lattice just described.

\section{Dissociation of Cartilage Cell and Osteoblast}

The fact that the cartilage cells continue to multiply while the osteoblasts of the shaft cease to 
be able to function, which, as just stated, is the basis for the formation of the growth retardation lattice, raises the general topic of dissociation between the growth activities of the cartilage and of the bone cells, a subject already discussed by Follis and me (Follis and Park, 1952). Such dissociations occur in a whole variety of pathological conditions. Chondrodystrophy is a classic example of one kind. In it, the cartilage cells lack the power to grow-in reality they multiply but at much reduced rates and the rates vary in different places. The variation in different parts of the epiphyseal cartilage is responsible for the curvatures produced in the shaft; the shafts are propagated bent like the sabre shin in rickets (Park, 1932) or the incisor teeth of rodents. The defect in this disease is primordial; the stuff of growth is not there. But there is nothing wrong with the osteoblasts, so that the shafts become thick. In osteogenesis imperfecta the reverse is the case; the cartilage retains its growth power, while the osteoblasts are able to produce only small quantities of highly defective matrix, as shown by the interesting studies by Follis (1952). The failure of the osteoblasts to produce normal amounts of matrix is shown in the finished bone by the closeness of the osteocytes to each other; the matrix formation was not adequate to spread them. Again here the defect is in the primordium but this time in osteoblastic function. In beautiful experiments of years ago, Wolbach (1933) and Wolbach and Howe (1926) showed that in scurvy the osteoblast has lost the power to form collagen, and Follis (1951) has carried knowledge still further by demonstrating that in the absence of vitamin $\mathrm{C}$, additional to failure in collagen production, the osteoblasts exhibited a decreased cytochrome oxidase and phosphatase activity and a reduction in ribosenucleic acid content as well. Thus, in scurvy new bone cannot form, but the cartilage continues to grow, for a time at least, and keeps forming calcified lattice which the shaft is powerless to take unto itself and transform into bone and equally powerless to destroy. Scurvy is a classical example of acquired dissociation, the cartilage cell retaining its growth power and the osteoblasts in complete functional failure. In congenital syphilis there is a similar dissociation, for the cartilage continues to grow but the osteoblasts in the spirochaetal areas are inactivated both in respect to their building and destroying function, so that the cartilage lattice develops at the end of the shaft by the same accumulative process as in scurvy or the growth retardation lattice just described. In experimental studies on vitamin A deficiency Wolbach (Wolbach, 1947; Wolbach and Bessey, 1941) noted a specific acquired dissociation characterized by complete growth cessa- tion of the cartilage, while appositional bone formation by the osteoblasts continued. Wolbach wrote:

'My own studies had convinced me several years ago, that, after endochondral bone formation had ceased in vitamin-A-deficient animals, appositional bone formation continued, and in certain locations apparently became excessive. Restudy of bones from vitamin-A-deficient animals, in the light of information obtained from the study of growth patterns of normal bones and of bones of animals which had been given greatly excessive amounts of vitamin $\mathrm{A}$, has made clear the fact that appositional bone formation in the A-deficient animals continues, until inanition supervenes, in strict conformity to normal growth patterns, both as to situation and rate.'*

I might cite other examples, but what I wish to bring out is that in our studies of children who have died from severe illness Follis and I have frequently encountered a dissociation of an entirely non-specific nature, depending presumably on nutritional failures of a general kind. In few instances it has been the cartilage cell which has been especially affected, either ceasing to grow or multiplying at a diminished rate, while the osteoblasts retained their function better and were able to thicken the trabeculae already formed. This kind of dissociation, cartilage cell failure and retention of osteoblastic activity, is the normal condition when growth of the cartilage comes to an end physiologically, that just preceding closure of an epiphysis, while osteoblastic action continues as long as life lasts. But far more commonly in our studies it has been the osteoblast which has lost its constructive vitality, while the cartilage cell has been able to continue linear growth.

\section{The Cause of these Dissociations}

The cartilage cell and the osteoblast differ in a variety of ways. That they differ metabolically is illustrated by the facts already mentioned, that under vitamin A deficiency cartilage growth stops while bone formation continues and that under vitamin $\mathrm{C}$ deficiency the osteoblasts cannot form bone, while the cartilage cell is able to produce cartilage. Both kinds of cells seem equally under the influence of the growth hormone, but the parathyroid hormone, while affecting the osteoblasts, leaves the cartilage cell alone. The cartilage cell growth is influenced by mechanical stimuli but only to a slight extent as compared to the osteoblasts whose activity is turned toward formation or destruction like a ship by its rudder. Witness the remodelling of bone after fracture or after the bending caused by rickets, when the components structurally useless are removed as

\footnotetext{
* Wolbach, 15b, p. 181.
} 
fast as new bone formation is required. In other words, the osteoblast presides over the bone mass, continually adjusting it to the mechanical needs. Moreover, it is probable that the osteoblasts are the guardians of the great calcium reservoir in the skeleton. The duty of the cartilage cell in contrast seems to be limited to the single track of growth. The stress and strain factors in the regulation of osteoblastic activity must be particularly important, and in this regard one must bear in mind that the sick child, more or less immobilized in bed, is deprived of the stimuli which keep formation active, and that the bone mass is in process of reduction to the lesser needs of existence in bed.

Of course there is abundant evidence for the dependence of bone growth on the blood supply. Pease (1952) has recently summarized the literature in connexion with his own experiments, showing that a whole variety of conditions, which have the common factor of increasing the circulation, stimulate longitudinal growth. Reduction in the circulation from local or systemic causes could undoubtedly produce the regressive changes in both cartilage and shaft caused by illness, but in that case the changes should correspond. We suggest that the regulating factor which determines the growth of the epiphyseal cartilage is its nutrient supply, but that the osteoblasts, within normal circulatory limits, are under the direct control of a variety of factors, as numerous as their functions, which vary according to the body's needs at the moment. These considerations perhaps make the dissociation between the two kinds of cells less to be wondered at, but we realize that the teleological thinking does not offer any real explanation of the cause.

\section{Significance of the Growth Disturbances of Cartilage and Shaft}

The slowing of growth of the cartilage and the regressive changes in the shaft mean that under severe illness the organism as a whole is freed, largely, if not entirely, from the load of supporting growth in a large area; in crisis the skeleton eliminates itself or is eliminated from the body economy. The cellular changes which we have described in the bone are visible there because of its peculiarly open structure and its durability. It would be interesting to know if in emergency other tissues of the body, not essential to keeping alive, behave similarly.

\section{Summary}

I have demonstrated the growth pattern characteristic of health in the bone of the young child, not too easy to determine, and its modification under the stress of severe illness and shown that in times of prosperity bone-forming cells grow big and crowd the bone surfaces and are producing bone rapidly, but in times of severe illness they give up their activity, shrink and revert to other forms and disappear until the trabeculae look almost bare; the picture of extreme cellular activity in a few days changes to an almost complete inertia. The study has made clear that one must think of bone, in the early period of life certainly, not as an inert tissue, but as a buzzing hive of activity and also as a highly sensitive tissue, adapting itself rapidly to variations in the general well being and so constituted that in times of special strain it can relinquish its growth activity entirely and relieve the general economy by elimination of that added burden.

One must also think of growth in bone as not necessarily a continuous process but rather as one linked closely to the general state and subject to exacerbations and checks and rises and falls in rate to correspond with variations in the latter. Finally, I have shown, with reference to growth under environmental conditions in common, that the cartilage cell and the osteoblast can behave very differently, even oppositely, and in pointing out the differences I have tried to make clear what the lines of arrested growth of the cartilage and the growth retardation lattice, both of which are visible in the $x$-ray film, actually were and how each came into being and how they both depended on opposite combinations of divergencies in the growth operation of the two tissues.

\section{REFERENCES}

Benjamin, H. R., Gordon, H. and Marples, E. (1943). Amer. J. Dis. Child., 65, 412. Bloom, W., Bloom, M. A. and McLean, F. C. (1941). Anat. Rec.,

Clifford. S. H. and Weller, K. F. (1948). Pediatrics, 1, 505.

Follis, R. H. (1949). Bull. Johns Hopk. Hosp., 85, 360. (1951). Ibid., 89, 9.

(1952). J. Pediat., 41, 713

— and Park, E. A. (1952). Amer. J. Roentgenol., 68, 709. 480 and Jackson, D. (1952). Bull. Johns Hopk. Hosp., 91,

Gordon, H. H. and McNamara, H. (1941). Amer. J. Dis. Child., 62, 328

Gutman, A. B. and Yu, T. F. (1950). Metabolic Interrelations. Trans. 2nd Conf., 1950, Josiah Macy, Jr. Foundation, New York.

Harris, H. A. (1933). Bone Growth in Health and Disease London.

Kölliker, A. (1873). Die normale Resorption des Knochengewebes, und ihre Bedeutung für die Entsichung der typischen Knochenformen. Leipzig.

Lacroix, P. (1951). The Organization of Bones. London.

Park, E. A. (1932). Canad. med. Ass. J., 26, 3.

— and Richter, C. P. (1953). Bull. Johns Hopk. Hosp., 93, 234.

Pease, C. N. (1952). J. Bone Jt Surg., 34A, 1.

Wolbach, S. B. (1933). Amer. J. Path. 9, 689

- (1947). J. Bone Jt Surg., 29, 171.

and Bessey, O. A. (1941). Arch. Path., Chicago, 32, 689.

and Howe, P. R. (1926). Ibid., 1, 1. Wright, S. W., Filer, L. J. and Mason, K. E. (1951). Pediatrics, 7, 